\title{
Haloperoxidases de vanádio - enzimas muito versáteis
}

\section{Resumo}

Haloperoxidases de vanádio (V-HPO) são enzimas que catalisam uma diversidade substancial de reacções, desde a halogenação à sulfoxidação, sendo operacionais numa gama alargada de temperaturas e em misturas solvente orgânico-água. Na sua forma apo apresentam actividade de fosfatases. A inversa é, nalguns casos, verdadeira
- certos fosfatases, quando incubados com vanadato, apresentam actividade halogenante e/ou sulfoxidante. Embora o grau de homologia global existente entre os vários enzimas não seja muito elevado, a excepção é o centro activo cuja semelhança é muito grande. A reactividade destes enzimas é discutida com base em dados estruturais e em dados de mutações, efectuadas nos centros activos e nas suas vizinhanças.

\section{Introdução}

Haloperoxidases são enzimas produzidos por muitos sistemas biológicos que catalisam a oxidação de halogenetos (cloreto, brometo e iodeto) pelo peróxido de hidrogénio.

Na alga Penicillus capitatus foi encontrado o primeiro haloperoxidase de que há registo para o meio marinho, verificando tratar-se de um enzima hémico [1]. Mas, outros haloperoxidases tinham sido já descobertos, como por exemplo, no fungo Caldariomyces fumago [2], nos leucócitos [3], nos eosinófilos [4] e no leite [5].

Durante muito tempo pensou-se que todos os haloperoxidases eram enzimas hémicos [6] mas, no início dos anos 80, foi descoberto o primeiro haloperoxidase de vanádio na alga castanha Ascophyllum nodosum [7]. Seguidamente, foi demonstrado que o vanádio se encontrava no grupo prostético dos haloperoxidases de várias algas castanhas
[8-10]. Desde então a presença de haloperoxidases de vanádio foi detectada em outros organismos como nas algas vermelhas, Ceramium rubrum [11], Corallina pilulifera [12] e em fungos terrestres como Curvularia inaequalis [13].

$\mathrm{Na}$ costa portuguesa várias espécies de algas da ordem Laminariales, (Saccorhiza polyschides, Phyllariopsis brevipes, Laminaria saccharina, Laminaria hyperborea, Laminaria ochroleuca) e da ordem Fucales (Pelvetia canaliculata, A. nodosum) apresentam haloperoxidases de vanádio [14-17].

Os haloperoxidases de vanádio apresentam-se frequentemente como agregados de monómeros de massa molecular 6570 kDa [13] ou em estruturas homo-diméricas, como o haloperoxidase de vanádio da $A$. nodosum, em que a massa molecular é cerca de 120 kDa [18] ou ainda em estruturas dodecaméricas de iguais subunidades, como é o caso do haloperoxidase da C. officinalis [19].
Uma característica interessante destes enzimas é a sua notável estabilidade operacional. Estes enzimas podem reter a sua actividade, durante cerca de um mês, quando armazenados em $60 \%$ de metanol, etanol e isopropanol e permanecem totalmente activos quando expostos a temperaturas até $70^{\circ} \mathrm{C}$ por períodos de tempo relativamente longos $[8,20]$. No nosso laboratório observámos que estes enzimas, quando liofilizados, permaneceram mais de 6 meses, à temperatura ambiente, sem perda de actividade.

Os haloperoxidases podem distinguir-se, com base no seu grupo prostético, em haloperoxidases hémicos, que contêm o ferro ligado a uma protoporfirina IX ou derivado e os haloperoxidases não hémicos onde se incluem os haloperoxidases de vanádio [21]. Uma propriedade geral dos haloperoxidases de vanádio é o facto de o vanádio, na forma de oxoanião, poder ser removido por diálise contra EDTA, em tampão citrato-fosfato a

1-Centro de Química e Bioquímica - Departamento de Química e Bioquímica da Faculdade de Ciências da Universidade de Lisboa, Edifício C8, Campo Grande, 1749-016 Lisboa, Portugal

2-Instituto de Tecnologia Biomédica, Faculdade de Medicina Dentária da Universidade de Lisboa, 1649-003 Lisboa, Portugal 
baixo $\mathrm{pH}(3,8)$, levando à inactivação do enzima. A actividade enzimática pode, contudo, ser completamente restabelecida por adição de vanadato [22].

Os haloperoxidases de vanádio podem ser também classificados segundo o halogeneto mais electronegativo que são capazes de oxidar. Assim, um cloroperoxidase pode oxidar cloreto, brometo e iodeto, enquanto que um bromoperoxidase pode oxidar brometo ou iodeto e um iodoperoxidase pode oxidar apenas o iodeto, embora a distinção entre estas subclasses não seja, por vezes, muito clara.

\section{Estrutura dos haloperoxidases de vanádio}

Até ao momento foram publicadas as estruturas de raios- $X$ de apenas três haloperoxidases contendo vanádio.

\section{Bromoperoxidase de vanádio (V-BPO) da alga $\boldsymbol{A}$. nodosum}

As estruturas primária e tridimensional do V-BPO (EC 1.1.11.X) extraído da alga A. nodosum revelaram tratar-se de uma proteína homo-dimérica com 1111 aminoácidos de forma aproximadamente elipsoidal. Os dois monómeros estão ligados por duas pontes dissulfureto intermoleculares. A sequência primária (SwissProt access code 1Q19) do V-BPO foi estabelecida combinando os resultados de sequenciação de DNA e de proteína com a interpretação dos mapas de densidade electrónica. O holoenzima contém um átomo de vanádio, numa estrutura de bipirâmide trigonal, em cada um dos dois centros activos (Figura 1) [23].

No estado nativo o átomo de vanádio encontra-se no centro de uma bipirâmide trigonal, com três átomos de oxigénio coordenados no plano equatorial; as cargas negativas destes átomos de oxigénio são compensadas por ligações de hidrogénio a vários aminoácidos da vizinhança, positivamente carregados e com carácter hidrofílico, os quais incluem Lys ${ }^{341}, \operatorname{Arg}^{349}$, Ser $^{416}$, Gly$^{471}$ e $\mathrm{Arg}^{480}$. O oxigénio axial forma uma ligação de hidrogénio com o átomo $N^{\delta 1}$ da histidina distal $\mathrm{His}^{418}$ e uma ligação covalente com o átomo $\mathrm{N}^{\delta 2}$ da His ${ }^{486}$.

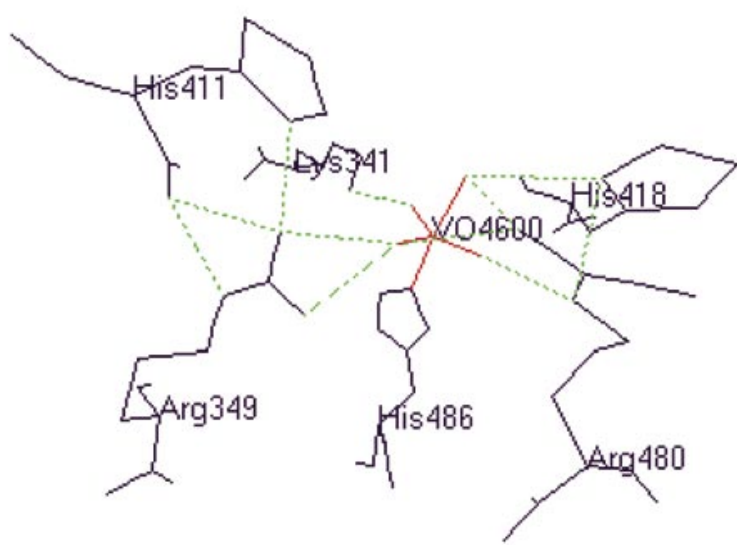

Figura 1 Estrutura do centro activo do bromoperoxidase de vanádio (V-BPO) da alga A. nodosum [23]. Linhas verdes a tracejado indicam ligações de hidrogénio. A figura foi preparada e modelada utilizando o programa Swiss PDB Viewer ${ }^{\circledR}$.

Estes resultados estão em concordância com os dados espectroscópicos e cinéticos que estabelecem a presença de um resíduo de histidina no centro activo do V-BPO [24].

A estrutura do V-BPO tem ainda uma segunda histidina próxima do centro activo $\left(\mathrm{His}^{411}\right)$ a qual, através de reacções de protonação/desprotonação modifica as propriedades catalíticas do enzima.

Locais de ligação específica para substratos orgânicos aromáticos (como o indole ou monoclorodimedona, por exemplo) ou para halogenetos, não são visíveis nesta estrutura.

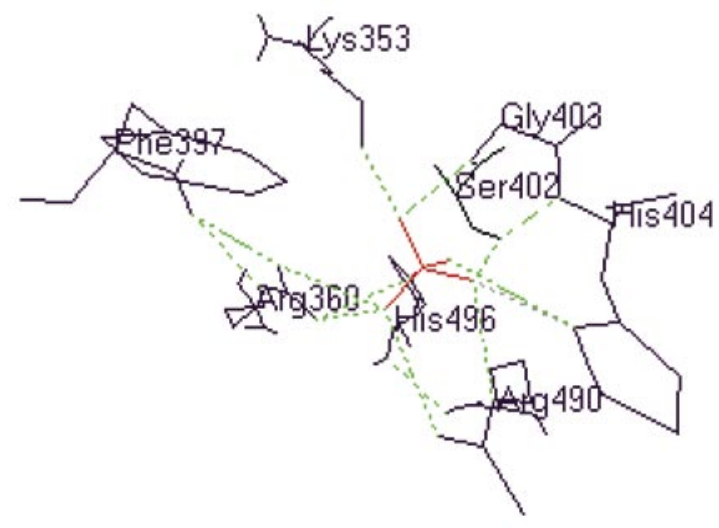

Figura 2 Representação esquemática do centro activo do cloroperoxidase de vanádio (V-CPO) do fungo C. inaequalis [30]. Linhas verdes a tracejado indicam ligações de hidrogénio. A figura foi preparada e modelada utilizando o programa Swiss PDB Viewer ${ }^{\circledR}$.

\section{Cloroperoxidase de vanádio (V-CPO) do fungo $C$. inaequalis}

O gene que codifica o V-CPO de C. inaequalis foi sequenciado, correspondendo a uma proteína de 609 aminoácidos. Tanto a estrutura primária [25] como a estrutura de raios-X do $\mathrm{V}$-CPO de $C$. inaequalis foram determinadas [26].

A forma global deste V-CPO monomérico é cilíndrica. 0 empacotamento compacto das hélices resulta num efeito hidrofóbico que é, provavelmente, o maior responsável pela estabilidade deste enzima.

Da estrutura cristalina da cloroperoxidase de $C$. inaequalis [26] verifica-se que o 


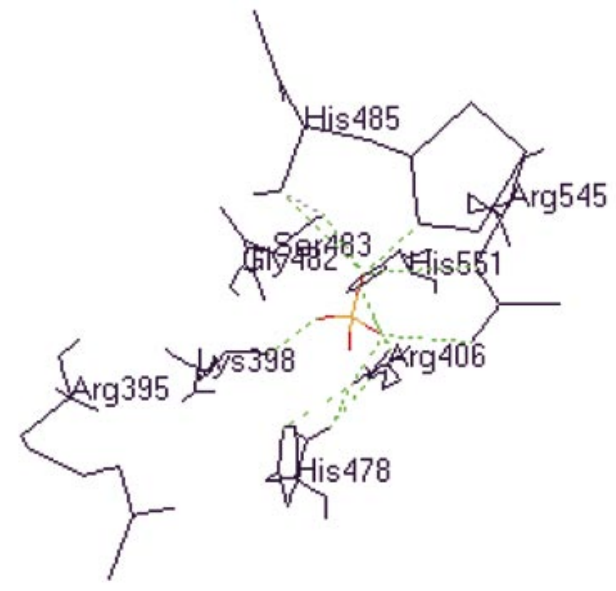

Figura 3 Estrutura do centro activo de uma das subunidades do bromoperoxidase de vanádio (V-BPO) da alga C. officinalis [23]. Linhas a verde e a tracejado indicam ligações de hidrogénio. A figura foi preparada e modelada utilizando o programa Swiss PDB Viewer ${ }^{\circledR}$. ião vanadato está ligado covalentemente ao átomo $\mathrm{N}^{\delta 2}$ da histidina proximal (His $\left.{ }^{496}\right)$, na posição apical, formando uma estrutura de bipirâmide trigonal. A carga negativa dos átomos de oxigénio é compensada, também aqui, pelos resíduos de carga positiva dos aminoácidos da vizinhança, que incluem Lys ${ }^{353}$, Arg $^{360}$, Ser $^{402,}$ Gly ${ }^{403}$ e Arg ${ }^{490}$ (Fig. 2).

O quarto oxigénio situa-se no vértice superior da pirâmide, na forma de grupo hidroxilo, o qual forma uma ligação de hidrogénio com o átomo de azoto $N \delta 1$ da $\mathrm{His}^{404}$ distal.

\section{Bromoperoxidase de vanádio da alga C. officinalis}

O bromoperoxidase da alga $C$. officinalis apresenta-se numa forma dodecamérica, com doze subunidades idênticas, num arranjo invulgar de simetria, com uma cavidade central formada pelos átomos de azoto terminais de cada subunidade [27]. A estrutura do centro activo de cada subunidade encontra-se na Figura 3. As subunidades, por sua vez, interactuam duas a duas, com um enrolamento e uma organização semeIhantes aos dímeros do enzima da alga A. nodosum, com o qual tem cerca de $33 \%$ de homologia.

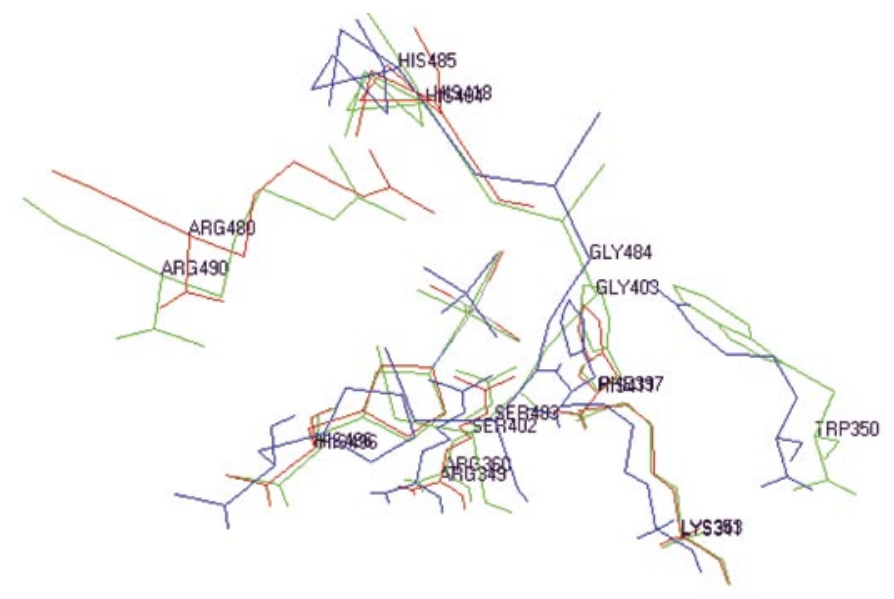

Figura 4 Sobreposição da estrutura dos centros activos dos três haloperoxidases de vanádio: Verde - C. ineaqualis; Vermelho - A. nodosum; Azul - C. officinalis. A figura foi preparada e modelada utilizando o programa Swiss PDB Viewer ${ }^{\circledR}$.

\section{Comparação do centro activo das haloperoxidases vanádio - aproximação catalítica}

Embora a comparação das estruturas tridimensionais do V-CPO do fungo $C$. inaequalis e das $\mathrm{V}$-BPO das algas $A$. nodosum e $C$. officinalis (Figura 4), revele que os centros activos são estruturalmente idênticos, a homologia entre as sequências dos VBPO e do V-CPO é baixa (aproximadamente 21.5\%).

$\mathrm{Na}$ estrutura do centro activo do V-BPO da $A$. nodosum, o resíduo catalítico $\mathrm{His}^{418}$ corresponde à His ${ }^{404}$ no $\mathrm{V}$-CPO. Uma segunda histidina $\left(\mathrm{His}^{411}\right)$, próxima do centro activo do V-BPO da A. nodosum pode alterar o potencial redox do intermediário peroxo, por reacções de protonação/desprotonação. Este resíduo $\left(\mathrm{His}^{411}\right)$ está também envolvido na estabilização dos átomos de oxigénio do grupo prostético, mas não se encontra no V-CPO. Por outro lado, o aminoácido $P^{397}$ encontra-se exclusivamente no V-CPO. Colocou-se a hipótese, de que esta diferença nos aminoácidos, possa ser responsável pela diferença na actividade de oxidação observada [28], uma vez que as cadeias laterais hidrofóbicas de $\operatorname{Trp}^{350}$ e Phe ${ }^{397}$ no V-CPO podem fornecer um ambiente estabilizador dos halogenetos e actuar como um ponto de ancoragem para estes. Também o resíduo $\mathrm{Ser}^{402}$, presente exclusivamente no V-CPO da $C$. inaequelis, na proximidade do grupo prostético, é um candidato provável para este efeito.

Nas três estruturas cristalográficas de V-HPO publicadas, todos os resíduos envolvidos na ligação ao vanadato se mantêm, mas apenas cinco dos 17 resíduos que delimitam a cavidade do centro activo são mantidos entre os dois V-BPO e nenhum destes cinco resíduos se encontra estruturalmente conservado no V-CPO. Nos V-BPO existe uma His ${ }^{478}$ que está localizada na posição da $\mathrm{Phe}^{397}$ que é proposta para a ligação do cloreto ao centro activo da V-CPO. Esta substituição, em conjunto com a ligação de hidrogénio da $\mathrm{Asp}^{333}\left(\mathrm{Asp}^{270}\right)$ à $\mathrm{His}^{485}$ $\left(\mathrm{His}^{418}\right.$ ) constitui a base da explicação para a preferência do brometo face ao cloreto. Na verdade, estes dois resíduos estão conservados nas V-BPO. 
Os resíduos envolvidos na ligação do vanadato estão conservados nestes três haloperoxidases. Contudo, a maioria dos outros resíduos que formam a cavidade do centro activo são diferentes, o que se pode traduzir em diferenças de especificidade de substratos e estereo-selectividade das reacções catalisadas por estes enzimas.

\section{Relação evolutiva entre V-HPO e fosfatases}

Apesar da homologia entre o V-BPO de A. nodosum e o V-CPO de $C$. inaequalis ser muito baixa, há alguns resíduos, em três domínios específicos, que são também conservados em pelo menos duas classes de fosfatases: os fosfatases de membrana e os fosfatases ácidos segregados [29].

A estrutura cristalina e as sequências primárias do fosfatase ácido não específico de Escherichia blattae [30] e do V-CPO de C. inaequalis [25] mostram uma grande similaridade nos centros activos, pondo a hipótese de os haloperoxidases de vanádio e as várias classes de fosfatases ácidos terem evoluído de um mesmo antecedente [29]. Foi surpreendente descobrir que a forma apo do cloroperoxidase de vanádio apresenta actividade catalítica como fosfatase, mediando a hidrólise do $p$-nitrofenilfosfato [29]. Os dados cinéticos da reacção de fosfatase catalizada pelo apocloroperoxidase de vanádio apontam para um mecanismo com a formação de um intermediário enzimático fosforilado. É importante salientar que os fosfatases ácidos, com um grau de homologia elevado com o V-CPO de $C$. inaequalis, nos quais foi incorporado vanadato, revelam actividade de oxidação do brometo e promovem reacções enantio-selectivas de transferência de oxigénio, com bons rendimentos [31, 32]. Curiosamente, a incorporação de vanadato num fitase extraída do Aspergillus ficuum e num fosfatase ácido extraído do germén de trigo, não homólogos ao V-HPO, permitem a estes enzimas catalisarem reacções de transferência enantio-selectiva de oxigénio [33].
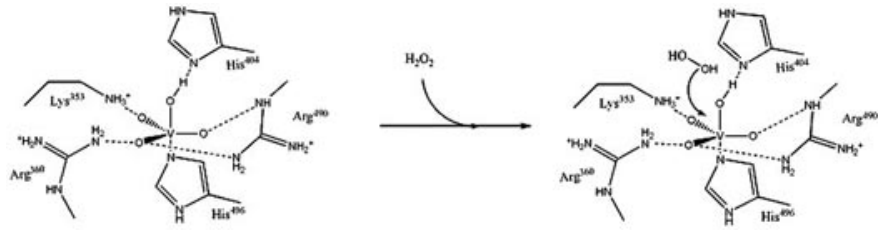

нго
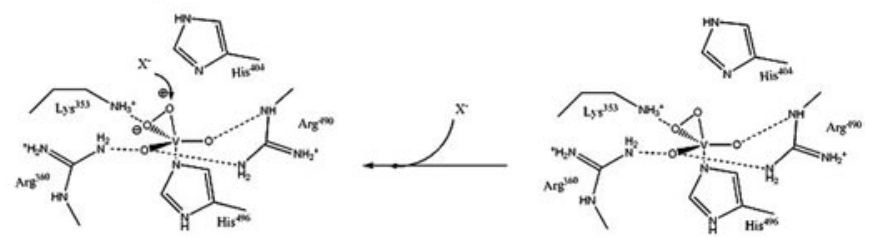

Figura 5 Esquema básico do mecanismo de oxidação do halogeneto por acção do cloroperoxidase de vanádio do fungo C. inaequalis [39].

\section{Aspectos mecanísticos das reacções de halogenação}

\section{Mecanismo catalítico geral das haloperoxidases}

Os haloperoxidases são enzimas que oxidam halogenetos a ácidos hipo-halosos, à custa do peróxido de hidrogénio (ou outro peróxido):

$\mathrm{X}^{-}+\mathrm{H}_{2} \mathrm{O}_{2}+\mathrm{H}^{+} \rightarrow \mathrm{HOX}+\mathrm{H}_{2} \mathrm{O}$

em que $X^{-}=\mathrm{Cl}^{-}, \mathrm{Br}^{-}, \mathrm{I}^{-}$(ou o pseudo halogeneto $\mathrm{SCN}^{-}$) [34].

Na presença do peróxido de hidrogénio, através de uma transferência de dois electrões, produz-se o intermediário ácido hipo-haloso, muito reactivo e que pode prosseguir a reacção, se estiver presente um aceitador nucleófilo (HA), para formar um vasto leque de compostos halogenados, como se esquematiza na seguinte reacção:

$\mathrm{HOX}+\mathrm{AH} \rightarrow \mathrm{AX}+\mathrm{H}_{2} \mathrm{O}$

$\mathrm{Na}$ ausência de um composto aceitador (HA), verifica-se um processo designado por disproporção assistida por halogeneto - o ácido hipo-haloso pode ser reduzido por um equivalente adicional de peróxido de hidrogénio, produzindo-se oxigénio molecular singleto [35, 36]

$\mathrm{HOX}+\mathrm{H}_{2} \mathrm{O}_{2} \rightarrow{ }^{1} \mathrm{O}_{2}+\mathrm{X}^{-}+\mathrm{H}^{+}+\mathrm{H}_{2} \mathrm{O}$
Apesar da diversidade de produtos halogenados formados, apenas três classes de enzimas halogenantes (haloperoxidases, halogenases dependentes de FAD 2 e metilo-transferases) parecem estar envolvidos na biossíntese de compostos orgânicos halogenados [37]. Pensa-se que a secreção destes metabolitos halogenados, que exibem muitas vezes uma actividade biológica considerável, pode estar ligada, directa ou indirectamente, aos mecanismos de defesa dos organismos [38].

O mecanismo proposto para a actuação catalítica dos haloperoxidases de vanádio é um mecanismo do tipo pingpong [35, 36], onde o primeiro substrato $\left(\mathrm{H}_{2} \mathrm{O}_{2}\right)$ se liga ao enzima, formando o peroxocomplexo com libertação do primeiro produto $\left(\mathrm{H}_{2} \mathrm{O}\right)$, antes da ligação e oxidação do segundo substrato ( $X^{-}=$ halogeneto). Embora os parâmetros cinéticos sejam diferentes, os estudos cinéticos com os vários enzimas parecem apontar para um mecanismo semelhante nas reacções de oxidação do halogeneto (cloreto e brometo) catalisadas pelas cloro- e bromoperoxidases de vanádio (Figura 5).

\section{Ciclo catalítico}

Uma característica interessante destes enzimas é o facto do estado de oxidação do vanádio se manter inalterado duran- 


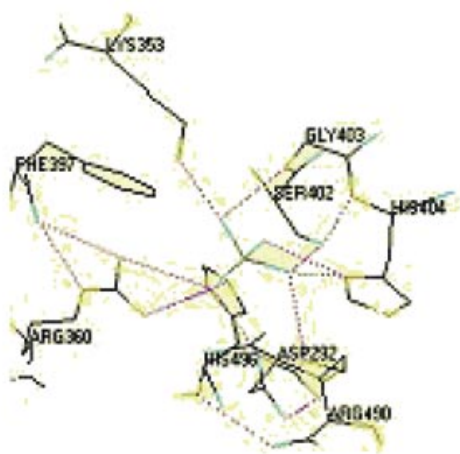

A

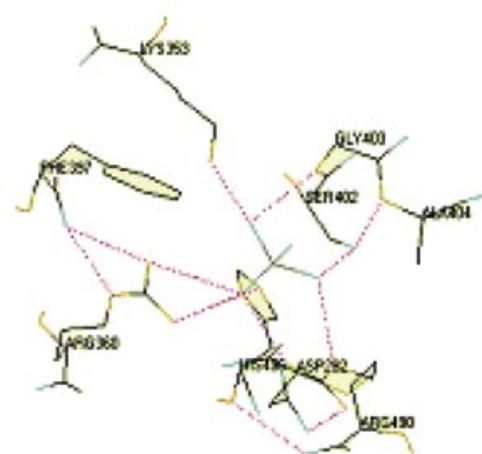

B

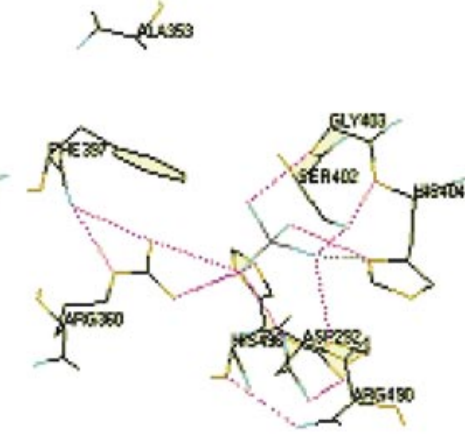

C

Figura 6 Comparação de algumas estrutura do centro activo: (A) cloroperoxidase de vanádio de C. inaequalis, (B) mutante V-CPO His 404Ala e (C) mutante Ly ${ }^{353} \mathrm{Ala}$. Linhas verdes a tracejado indicam ligações de hidrogénio. A figura foi preparada e modelada utilizando Swiss PDB Viewer ${ }^{\circledR}$

te todo o ciclo catalítico, ao contrário do que se verifica nas haloperoxidases hémicas.

Durante o ciclo catalítico e para o caso do V-CPO do C. inaequalis, o grupo prostético $\left(\mathrm{HVO}_{4}{ }^{3-}\right)$ está coordenado ao átomo $\mathrm{N}^{\delta 2}$ da $\mathrm{His}^{496}$. O peróxido liga-se, através dos dois átomos de oxigénio, ao átomo de vanádio. Assim, a geometria de coordenação em torno do átomo de vanádio passa a apresentar-se como uma pirâmide tetragonal distorcida. Inicialmente, foi proposto que o ião halogeneto se ligaria directamente ao vanadato; todavia o isolamento de um intermediário peroxo, aliado ao facto de que a ligação halogeneto-vanadato baixaria a sua nucleofilicidade, o que não se verifica, inviabilizaram esta hipótese [39].

As estruturas cristalinas, quer do enzima nativo quer do intermediário peroxo, no V-CPO, permitiram observar que o resíduo de aminoácido Lys ${ }^{353}$ está ligado por uma ponte de hidrogénio ao oxigénio do grupo prostético. Esta interacção parece ser muito importante para a coordenação do peróxido ao vanádio (V), labilizando um oxigénio equatorial do grupo prostético, facilitando, concomitantemente, o ataque nucleofílico do halogeneto, por polarização do peróxido coordenado [35, 36].
No V-BPO, o aminoácido correspondente $\left(\right.$ Lys $^{341}$ ) e o aminoácido adicional His ${ }^{411}$ podem estar envolvidos na ligação do peróxido ao vanádio e também na activação do peróxido coordenado [37].

\section{Mutagénese do V-CPO: uma aproximação ao mecanismo catalítico}

Afim de investigar a função específica dos aminoácidos do centro activo na actividade catalítica do V-CPO do fungo $C$. inaequalis, o enzima foi expresso através de um sistema heterólogo, na levedura Saccharomyces cerevesiae tendo sido produzidos mutantes, através de mutagénese dirigida [39, 40]. O cloroperoxidase de vanádio recombinante ( $r$-CPO) é reactivado por adição de vanadato e, quando comparado com o produzido naturalmente, apresenta um comportamento cinético semelhante. A estrutura cristalina mostra que a estrutura global do enzima permanece praticamente inalterada, particularmente, na vizinhança do centro activo [39].

Foram efectuadas experiências de mutagénese dirigida sobre diversos resíduos do centro activo, mutando-os para alaninas. Nesta cavidade, existe um grande número de interacções, essencialmente ligações de hidrogénio, que envolvem as cadeias laterais dos aminoácidos que a revestem. A substituição de resíduos de aminoácidos, com cadeias laterais gran- des, por grupos metilo de uma alanina, não parece provocar mudanças estruturais no local de ligação do vanádio, com excepção do mutante $\mathrm{His}^{404} \mathrm{Ala}$ que sofre algumas modificações estruturais (Fig. 6).

Demonstraram-se alguns efeitos estruturais, não muito significativos, nas mutações do resíduo $\operatorname{Arg}^{360}$, que está directamente envolvido na compensação da carga negativa de um dos oxigénios do vanadato, e também no resíduo Asp ${ }^{292}$, que está envolvido na formação de uma ponte salina com o aminoácido Arg ${ }^{490}$ e que, por sua vez, também interactua com o grupo prostético. Contudo, é relevante salientar que se verifica uma diminuição da actividade como haloperoxidase devido, provavelmente, à destabilização da rede de interações existentes na cavidade e que contribuem para a formação do peroxocomplexo.

O enzima perde igualmente a sua capacidade de oxidar o cloreto quando a $\mathrm{His}^{404}$ distal é substituída por uma alanina (mutante $\mathrm{His}^{404} \mathrm{Ala}$ ), mostrando assim que este resíduo desempenha um papel importante na estabilização do grupo hidroxilo apical [23].

No mutante His ${ }^{496} \mathrm{Ala}$, apesar de não se verificarem modificações estruturais, esta alteração resulta num enzima inactivo, devido à falta de capacidade do 
enzima ligar, neste caso, o grupo prostético, demonstrando assim a importância essencial da ligação do vanadato ao átomo $\mathrm{N}^{\delta 2}$ da $\mathrm{His}^{496}$.

Recentemente foram efectuados outros estudos utilizando mutagénese dirigida nos aminoácidos do centro activo Ser ${ }^{402}$ e Phe ${ }^{397}$ do cloroperoxidase da C. inaequalis.

Coloca-se então a hipótese de que estes aminoácidos possam marcar a diferença entre a capacidade oxidativa de halogenetos entre o V-BPO de $A$. nodosum e o V-CPO de $C$. inaequalis. A substituição de Phe $^{397}$ por uma histidina no V-CPO, utilizando a mutagénese dirigida, mostra que a actividade de cloração se mantém, embora diminuída. Apesar disso, este mutante ainda apresenta maior actividade de bromação do que o V-BPO de A. nodosum [41].

Por outro lado, estudos de EXAFS no VBPO sugerem que o resíduo Ser ${ }^{402}$ pode ter um papel importante na catálise, ligando o halogeneto, de forma covalente, ao grupo hidroxilo do aminoácido [42]. No entanto, resultados recentes demonstram que a mutação da $\mathrm{Ser}^{402}$ do V-CPO tem um efeito diminuto na actividade catalítica (resultados não publicados). A estrutura dos mutantes demonstra que o centro activo deste cloroperoxidase é uma matriz rígida, que fornece um local de ligação a um oxoanião. Embora a

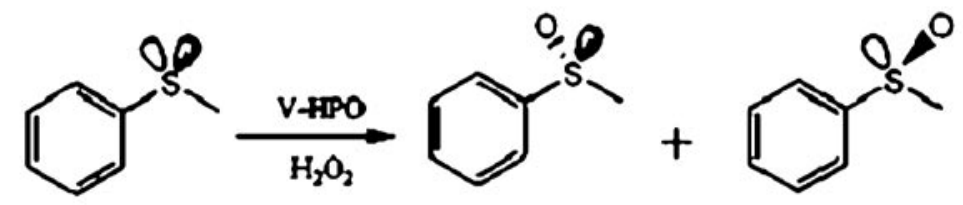

Figura 7 Reacção de transferência de oxigénio em sulfuretos, catalisada pelo V-HPO. Imagem concebida utilizando o progama ChemUltra $9.0^{\circledR}$.

capacidade de oxidação do cloreto ou brometo não possa ser especificamente atribuída a um resíduo aminoácido ela é, muito provavelmente, determinada por uma relação sinérgica entre vários resíduos e não por um só.

\section{Reacções de sulfoxidação}

$A$ adição de um átomo de oxigénio a um centro de enxofre, dentro de um composto orgânico formando uma ligação polarizada S-O, não só altera as propriedades físicas e químicas da molécula, mas pode também alterar profundamente a actividade biológica, a sua distribuição dentro do organismo e a sua forma de degradação.

Os sulfóxidos opticamente activos, são bastante conhecidos como ferramentas na síntese orgânica, devido à sua elevada indução assimétrica, o que pode ser atribuído a diferenças espaciais e estéreas entre os substituintes e a esta- bilidade conformacional dos sulfuretos [43-49].

A utilização de peroxocomplexos de vanádio (V) em reacções de oxidação tem sido investigada desde há várias décadas. Em 1986, foi sintetizado um peroxocomplexo, usando uma base de Schiff tridentada e opticamente activa, o qual catalisou a oxidação de um sulfureto de metilfenilo pelo hidroperóxido de t-butilo em diclorometano, conduzindo à formação do enantiómero $\mathrm{R}$ do correspondente sulfureto, com um excesso enantiomérico de 14\% [50]. Desde então, muitas outras reacções de oxidação, catalisadas por peroxocomplexos de vanádio (V), têm sido descritas [49-54].

O cloroperoxidase do fungo $C$. fumago, um haloperoxidase hémico, é, até à data, o haloperoxidase que produz a mais elevada enantioselectividade entre todos os haloperoxidases (hémi-

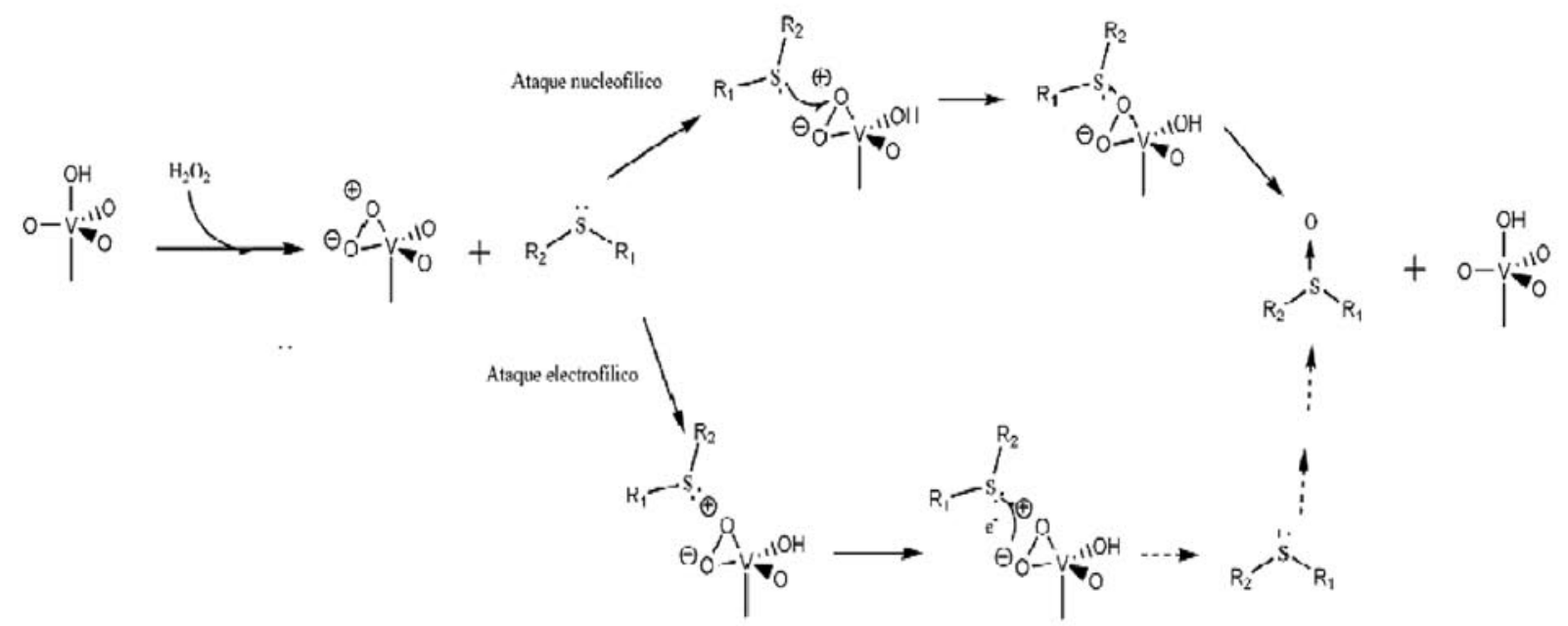

Figura 8 Proposta de possíveis mecanismos de transferência de oxigénio em peroxocomplexos de vanádio na presença de sulfuretos. R1 e R2 descrevem possiveis cadeias/grupos associadas ao átomo de enxofre. Imagem concebida utilizando o progama ChemUltra $9.0^{\circledR}$. 
cos e de vanádio) estudados até agora $[32,43,44,49,55,56]$.

Alguns autores, atribuem este facto, à presença de um resíduo do aminoácido
Foi já referida a relação evolucionária e semelhanças entre os centros activos dos fosfatases e haloperoxidases de vanádio. Para o confirmar, a um fitase extraído do Aspergillus ficuum, (não

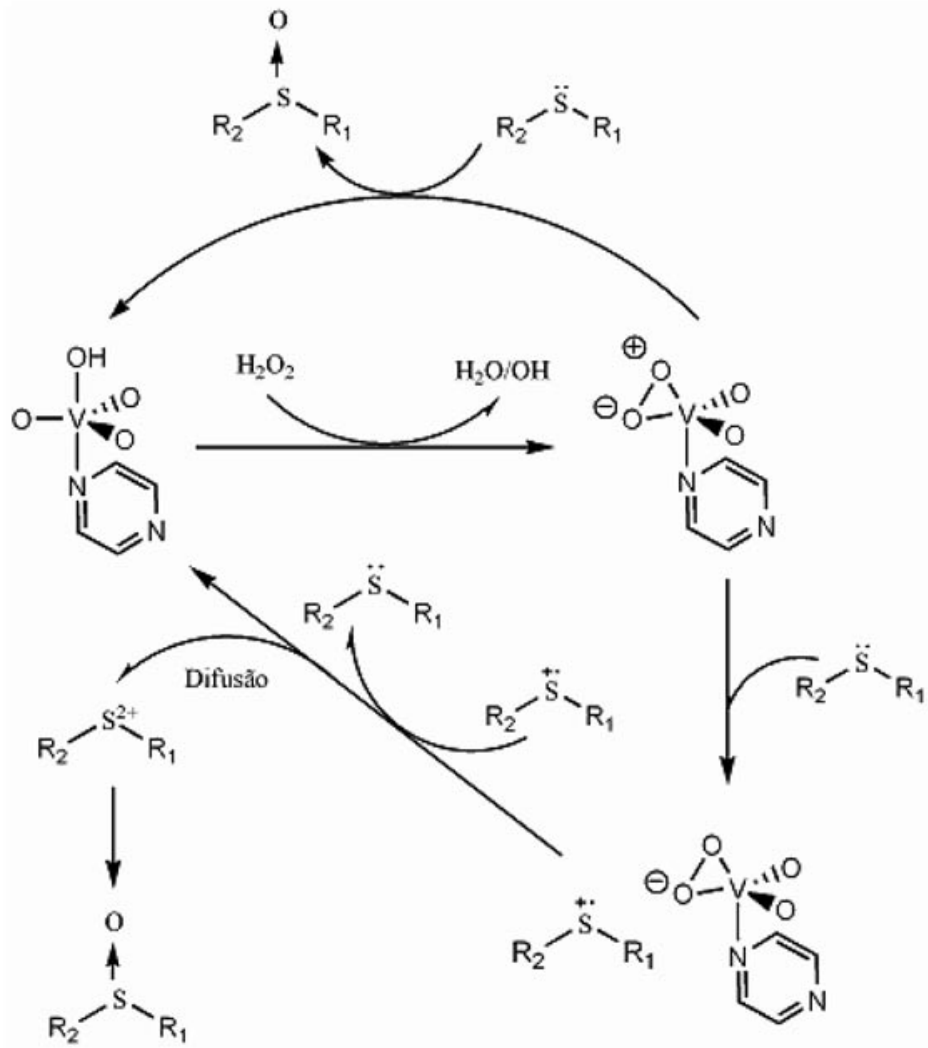

Figura 9 Ciclo catalítico da sulfoxidação catalisado pelos haloperoxidases de vanádio. Imagem concebida utilizando o programa ChemUltra $9.0^{\circledR}$

glutamato, na posição distal, no lugar do resíduo de histidina, que se encontra na maioria dos haloperoxidases, quer hémicos, quer de vanádio [44].

Ten Brink et al. demonstraram que 0 V-BPO extraída das algas $A$. nodosum e $C$. pilulifera e o V-CPO do fungo $C$. inaequalis catalisavam a sulfoxidação do p-metiltioanisole (Fig. 7) [32].

O V-BPO extraído da alga $A$. nodosum apresenta a maior taxa de enantioselectividade, produzindo o sulfóxido do tioanisole com um excesso enantiomérico de $91 \%$. Todavia, e embora a taxa de conversão para o V-CPO do $C$. inaequalis seja superior, o produto obtido é racémico [32]. homólogo dos V-HPO), foi incorporado vanádio no centro activo; este novo enzima é capaz de catalisar a sulfoxidação do tionanisole, embora com rendimento moderado e excesso enantiomérico reduzido [33].

No entanto, é importante salientar que, apesar do interesse existente nestas reacções, há ainda alguns impedimentos que condicionam a sua utilização, como por exemplo, a velocidade de reacção da maioria dos sulfuretos, biologicamente importantes, ser lenta devido, provavelmente, à baixa solubilidade dos sulfuretos em água ou ainda a existência de oxidação espontânea (não enzimática) dos sulfuretos com o peróxido de hidro- génio, que diminui consideravelmente o rendimento e o excesso enantiomérico dos sulfóxidos produzidos enzimaticamente $[32,48]$.

\section{Mecanismo catalítico da transferência de oxigénio}

$\mathrm{Na}$ sulfoxidação catalisada por peroxocomplexos inorgânicos de vanádio, a oxidação dos sulfuretos aos correspondentes sulfóxidos pode ser catalisada por um mecanismo de transferência de oxigénio que envolve um ataque electrofílico ou por um mecanismo de transferência de oxigénio nucleofílico (Figura 8) [57].

No mecanismo electrofílico, o processo de transferência de oxigénio envolve a transferência electrónica do peroxocomplexo de vanádio (V) activado, para um substrato electrofílico, sendo a reacção de oxidação mediada por um mecanismo radicalar [56, 58].

Na via nucleofílica, o substrato apresenta um comportamento nucleofílico, coordenando-se ao centro metálico, convertendo-o ao seu carácter original electrofílico, possibilitando o ataque nucleófilo pelo peróxido coordenado. Como os peroxocomplexos metálicos tendem a reagir através de um ataque nucleófilo, considerou-se que os sulfuretos se ligam ao peroxocomplexo de vanádio por um mecanismo de oxidação intramolecular, antes da transferência do oxigénio, favorecendo a via de transferência nucleofílica do oxigénio; no entanto, a coordenação do sulfureto parece pouco provável pois a sulfoxidação catalisada do vanádio (V) não é afectada pelo aumento da concentração do sulfureto ou por impedimentos estereoquímicos [32].

\section{Reacções enzimáticas de transferência de oxigénio}

A actuação dos enzimas é fortemente influenciada pela acessibilidade dos substratos ao centro activo e pela ocorrência de reacções não específicas de transferência electrónica, afectando deste modo a tão desejada enantioselectividade. A acessibilidade é regulada pela combinação de factores estéreos e electrónicos em consonância com a existência de factores de estabilização 
como, por exemplo, as pontes de hidrogénio entre os aminoácidos das cadeias laterais na vizinhança do centro activo e do oxoanião $\left(\mathrm{VO}_{4}{ }^{3-}\right)$.

Os mecanismos envolvidos nestas reacções de transferência de oxigénio diferem no caso da reacção envolver haloperoxidases hémicos ou de vanádio $[44,56,58,59]$.

Em termos mecanísticos, o primeiro passo da transferência de oxigénio nos processos de sulfoxidação pode ser comparada à formação do ácido hipohaloso: o peróxido liga-se ao átomo de vanádio lateralmente no plano equatorial, formando o peroxocomplexo [28]. Um dos átomos de oxigénio do peroxocomplexo tem uma carga parcial positiva e é vulnerável a um ataque nucleofílico pelo sulfureto (ou pelo halogeneto). É no segundo passo da reacção que os mecanismos divergem (Figura 8).

Para comprovar este mecanismo, foram realizadas experiências de marcação radioactiva do oxigénio e verificou-se que o oxigénio incorporado no sulfóxido provém, essencialmente, de peróxido marcado $\mathrm{H}_{2}{ }^{18} \mathrm{O}_{2}$ (95\%), no caso do V-BPO de $A$. nodosum. A mesma experiência realizada com o cloroperoxidase de $C$. inaequalis demonstrou que o oxigénio incorporado provém da água (95\%), o que parece confirmar que este cloroperoxidase tem caraterísticas intermédias entre os haloperoxidases de vanádio e os haloperoxidases hémicos [56].

A oxidação de substratos, como halogenetos e sulfuretos, é descrita, para os haloperoxidases de vanádio, como um mecanismo de transferência de dois electrões enquanto que para os haloperoxidases hémicos a catálise da reacção de oxidação é feita através de um mecanismo monoelectrónico, em que o enzima, sob a forma de peroxocomplexo, retira um electrão ao sulfureto, oxidando-o e fomando um sulfureto radicalar catiónico que é libertado para a solução (Figura 9).

A formação de um sulfureto radicalar foi já demonstrado para as reacções catalisadas por lactoperoxidase [33, 44].

Este radical difunde-se e reage com outras moléculas levando à formação de

Tabela I Reações de sulfoxidação do tioanisole catalisadas por vários enzimas - excesso enantiomérico (ee) e rendimento.

\begin{tabular}{lcc}
\hline Haloperoxidase & e.e. $(\%)$ & Rendimento \\
\hline Ascophyllum nodosum & $85(\mathrm{R})$ & 55 \\
Coralina officinalis & $91(\mathrm{~S})$ & 84 \\
Coralina pilulifera & $55(\mathrm{~S})$ & 18 \\
Caldariomyces fumago & $>99(\mathrm{R})$ & 99.5 \\
\hline
\end{tabular}

uma mistura racémica que é independente da concentração do enzima e do substrato.

Este passo tem lugar fora do centro activo e esta molécula radicalar reage, de forma aleatória, ora com outra molécula idêntica ora com moléculas de solvente ou oxigénio.

Mas, é igualmente possível que duas moléculas de sulfureto radicalar catiónicas possam reagir entre si para formar um sulfureto dicatiónico e uma molécula de sulfureto, sendo a reacção entre o dicatião e o solvente, neste caso a água, bastante provável [56, 59].

Apesar da existência de um mecanismo para a reacção de transferência de oxigénio, ainda não há uma justificação plenamente aceite para o facto dos $\mathrm{V}$-BPO das algas $A$. nodosum e $C$. officinallis catalisarem enantio-selectivamente a formação do enantiómero R (superior a 91\% [32] e 95\% [43], respectivamente), enquanto que o VBPO da C. pilulifera apenas apresenta um excesso enantiomérico de 55\% do enantiómero S (Tabela I) [32].

Este facto poderá dever-se a um baixo turnover, que traz como consequência um aumento da reacção não enzimática entre o peróxido de hidrogénio e o sulfureto levando a uma diminuição considerável da enantio-selectividade.

\section{Conclusão}

Os haloperoxidases de vanádio são os catalisadores da oxidação de halogenetos mais eficientes até à data encontrados. 0 mecanismo de catálise envolve a formação prévia de um peroxocomplexo.
As reacções são fortemente influenciadas pelos resíduos de aminoácidos do centro activo, que é muito semelhante nas três estruturas de haloperoxidases nativos até agora conhecidos. No entanto, a sua reactividade para alguns substratos, nomeadamente nas reacções de transferência de oxigénio, pode ser muito diversa, o que indicia um papel importante dos aminoácidos menos próximos do centro activo.

A homologia entre centros activos de alguns fosfatases e haloperoxidases abre também algumas hipóteses interessantes, em termos de mecanismos de regulação e biogénese, sobretudo de produtos halogenados com proveniência marinha.

Finalmente, a grande estabilidade e a reactividade destes compostos torna-os óptimos candidatos a biocatalisadores, em várias áreas farmacêuticas, em medicina nuclear e em oncomedicina.

\section{Bibliografia}

1. J. A. Manthey e L. P. Hager, Journal of Biological Chemistry 256 (1981) 11232-11238.

2. P. F. Hallenberg e L. P. Hager, Methods in Enzymology 52 (1978) 521-529.

3. G. C. Mills, Journal of Biological Chemistry 229 (1957) 189-197.

4. R. Wever, M. N. Hamera, R. S. Weening e D. Roos, European Journal of Biochemistry 108 (1980) 491-495.

5. M. Morrison, H. B. Hamilton e E. Stotz, Journal of Biological Chemistry 228 (1957) 767-776. 
6. S. Ohtaki, H. Nakagawa, M. Nakamura e I. Yamazaki, Journal of Biological Chemistry 260 (1985) 1387-1390.

7. H. Vilter, Phytochemistry 23 (1984) 13871390.

8. H. Plat, B. E. Krenne e R. Wever, Biochemistry Journal 248 (1987) 277-279.

9. E. De Boer, Y. Van Kooyk, M. G. M. Tromp, H. Plat e R. Wever, Biochimica and Biophysica Acta 869 (1986) 48-53.

10. B. E. Krenn, Y. Izumi, H. Yamada e R. Wever, Biochimica and Biophysica Acta 998 (1989) 63-68.

11. N. Itoh, Y. Izumi e H. Yamada, Biochemistry Biophysics Research Communications 131 (1985) 428-435.

12. N. Itoh, Y. Izumi e H. Yamada, Journal of Biological Chemistry 261 (1986) 5194-5200.

13. J.W. van Schijndel, E.G. Vollenbroek e R. Wever, Biochimica and Biophysica Acta 1161 (1993) 249-256.

14. M. Almeida, M. Humanes, J. A. Silva, R. Melo e J. J. R. Fraústo da Silva, 'Plant peroxidases: Biochemistry and physiology', em C. Obinger, U. Burner, R. Eberman, C. Penel, H. Greppin (eds), University of Geneva (1996) 146-152.

15. M. Almeida, M. Humanes, J. A. Silva, R. Melo, J. J. R. Fraústo da Silva, H. Vilter e R. W ever, Phytochemistry 48 (1998) 229-239.

16. G. Almeida, M. Humanes, J. A. Silva, R. Melo, J. J. R. Fraústo da Silva e R. Wever, Phytochemistry 54 (2000) 5-11.

17. M. Almeida, S. Filipe, M. Humanes, M. F. Maia, R. Melo, N. Severino, J. A. L. da Silva, J. J. R. Fraústo da Silva e R. Wever, Phytochemistry 57 (2001) 633-642

18. B. E. Krenn, H. Plat e R. Wever, Biochimica and Biophysica Acta 912 (1989) 287291.

19. C. Rush, A. Willetts, G. Davies, Z. Dauter, H. Watson e J. Littlechild, FEBS Letters 359 (1995) 244-246.

20. E. De Boer, H. Plat, M. G. M. Tromp, R. Wever, M. C. R. Franssen, H. C. van der Plas, E. M. Meijer e H. E. Schoemaker, Biotechnology and Bioengineering 30 (1987) 607-610.

21. H. Vilter, Biological Systems 31 (1995) 325-362.

22. R. Wever, B. E. Krenn, E. De Boer, H. Offenberg e H. Plat, Progress in Clinical Biological Research 274 (1988) 477-493.

23. M. Weyand, H.-J. Hecht, M. Kieß, M.-F. Liaud, H. Vilter e D. Schomburg, Journal of Molecular Biology 293 (1999) 595-611.
24. J. M. Arber, E. de Boer, C. D. Garner, S. S Hasnain e R. Wever, Biochemistry 28 (1989) 7968-7973.

25. L. H. Simons, P. Barnett, E. G. M. Vollenbroek, H. L. Dekker, A. O. Muijers, A. Messerschmidt e R. Wever, European Journal of Biochemistry 229 (1995) 566-574.

26. A. Messerschmidt e R. Wever, Proceedings of the National Academy of Sciences USA 93 (1996) 392-396.

27. M. N. Isupov, A. R. Dalby, A. A. Brindley, Y. Izumi, T. Tanabe, G. N. Murshudov e J. A. Littlechild, Journal of Molecular Biology 299 (2000) 1035-1049.

28. S. Macedo-Ribeiro, W. Hemrika, R. Renirie, R. Wever e A. Messerschmidt, Journal of Biological Inorganic Chemistry 4 (1999) 209-219.

29. W. Hemrika, R. Renirie, H. L. Dekker, P. Barnett e R. Wever, Proceedings of the National Academy of Sciences USA 94 (1997) 2145-2149.

30. K. Ishikawa, Y. Mihara, K. Gondoh, E. Suzuki e Y. Asano, EMBO Journal 19 (2000) 2412-2423.

31. N. Tanaka, V. Dumay, Q. Liao, A. J. Lange e R. Wever, European Journal of Biochemistry 269 (2002) 2162-2167.

32. H. B. Ten Brink, A. Tuynman, H. L. Dekker, W. Hemrika, Y. Izumi, T. Oshiro, H. E. Schoemaker e R. Wever, Inorganic Chemistry 37 (1998) 6780-6784.

33. F. Van de Velde, L. Konemann, F. Van Rantwijk e R. A. Sheldon, Biotechnology and Bioengineering 67 (2000) 87-96.

34. S. L. Neidleman e J. Geigert, Biohalogenation: Principles, Basic Rules and Applications, Ellis Horwood Limited, John Wiley \& Sons, New York, 1986.

35. R. R. Everett, H. S. Soedjak e A. Butler, Journal of Biological Chemistry 265 (1990) 15671- 15679.

36. H. S. Soedjak, J. V. Walker e A. Butler, Biochimica and Biophysica Acta 1079 (1991) 1-7.

37. M. C. R. Franssen, Biocatalysis 10 (1994) 87-111.

38. A. Butler e J. N. Carter-Franklin, Natural Product Reports 21 (2004) 180-188.

39. W. Hemrika, R. Renirie, S. Macedo-Ribeiro, A. Messerschmidt e R. Wever, Journal of Biological Chemistry 274 (1999) 2382023827.

40. R. Renirie, W. Hemrika e R. Wever Journal of Biological Chemistry 275 (2000) 11650- 11657.
41. M. Weyand, H. -J. Hecht, M. Kie, M. F. Liaud, H. Vilter e D. Schoumbourg, Journal of Molecular Biology 293 (1999) 595-611.

42. H. Dau, J. Dittmer, M. Epple, J. Hanss, E. Kiss, D. Redher, C. Schulzke e H. Vilter, FEBS Letters 457(1999) 237-240.

43. M. Andersson, A. Willets, S. Allenmark, Journal of Organic Chemistry 62 (1997) 8455-8458.

44. M. P. J. van Deurzen, F. van Rantwijk e R. A. Sheldon, Tetrahedron 53 (1997) 1318313220.

45. V. M. Dembitsky, Tetrahedron 59 (2003) 4701-4720.

46. S. Hu e J. S. Dordick, Journal of Organic Chemistry 67 (2002) 314-317.

47. A. G. J. Ligtenbarg, R. Hage e B. L. Feringa, Coordination Chemistry Reviews 237 (2003) 89- 101.

48. L. Dai e A. M. Klibanov, Biotechnology and Bioengineering 70 (2000) 353-357.

49. E. Baciochi, M. F. Gerini, P. J. Harvey, O. Lanzalunga e S. Mancinella, European Journal of Biochemistry 267 (2000) 2705-2710.

50. K. Nakajima, M. Kojima, K. Toriumi, K. Saito e J. Fujita, Bulletin of the Chemical Society of Japan 62 (1989) 760-776.

51. M. Picard, J. Gross, E. Lübbert, S. Tölzer, S. Krauss, K.-H. van Pée e A. Berkessel, Angewandte Chemie 109 (1997) 1245-1248.

52. A. H. Vetter e A. Berkessel, Tetrahedron Letters 39 (1998) 1741-1744.

53. A. K. Sharipov, Russian Journal of Applied Chemistry 76 (2003) 108-113.

54. S. Kobayashi, M. Nakano, T. Kimura, e P. A. Schaap, Biochemistry 30 (1987) 50195022.

55. S. G. Allenmark e M. A. Andersson, Chirality 10 (1998) 246-252.

56. H. B. Ten Brink, H. E. Schoemaker e R. Wever, European Journal of Biochemistry 268 (2001) 132-138.

57. F. Di Furia e G. Modena, Pure \& Applied Chemistry 54 (1982) 1853-1866.

58. H. B. Ten Brink, H. L. Dekker, H. E. Schoemaker e R. Wever, Journal of Inorganic Chemistry 30 (2000) 91-98.

59. A. Tuynman, M. K. S. Vink, H. L. Dekker, H. E. Schoemaker e R. Wever, European Journal of Biochemistry 258 (1998) 906-913. 


\section{Mystery SOLVED}

Gemini $^{\text {TM }}$ with new Twin ${ }^{\text {Ts }}$

(Two-In-One) Technology

provides $\mathrm{pH}$ 1-12 stability with

no sacrifice in performance.

Gemini is engineered to provide

unmatched performance

and column lifetime.
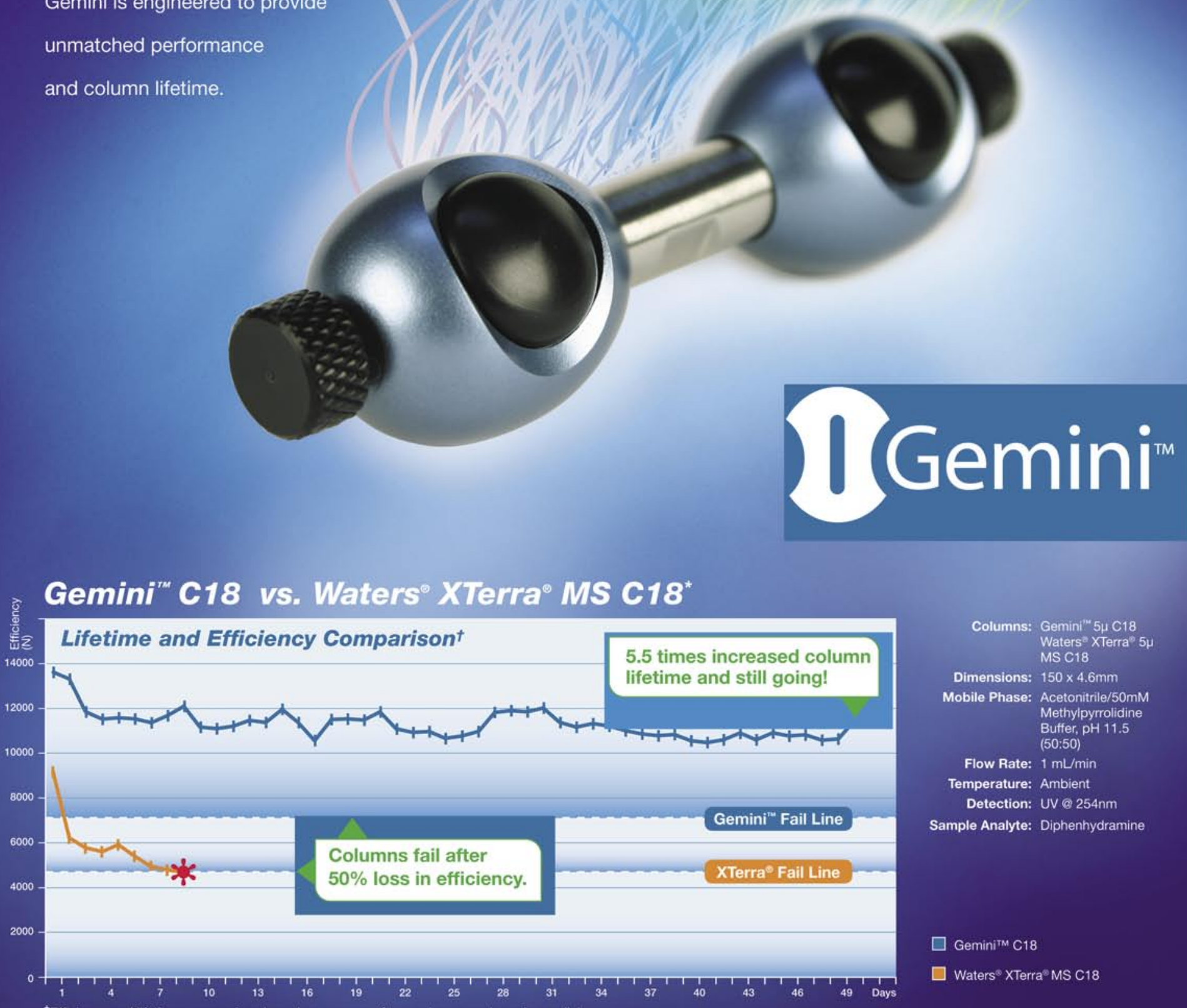

\section{Gemini' ${ }^{m} 18$ vs, Waters ${ }^{\circ}$ Terra $^{\circ}$ MS C18*}

\author{
Columns: Gemini"' $5 \mu \mathrm{C} 18$ \\ Waters" XTerra" $5 \mu$ \\ MS $\mathrm{C} 18$ \\ Dimensions: $150 \times 4.6 \mathrm{~mm}$ \\ Mobile Phase: Acetonitrile/50mM \\ Methylpyrrolidine \\ Buffer, $\mathrm{pH} 11.5$ \\ Bufier,
$(50: 50)$
}

Flow Rate: $1 \mathrm{~mL} / \mathrm{min}$

Temperature: Ambient

Detection: UV @ 254nm

Sample Analyte: Diphenhydramine $\square$ GeminiTM $\mathrm{C} 18$

$\square$ Waters $^{*}$ XTerra" MS C18

tEfficiency and lifetimes comparison based on average of two columns each run in parallel.

\section{Visit}

www.phenomenex.com/gemini

\section{Pphenomenex} (19) whwophenomenex:com

\begin{tabular}{|c|c|c|c|c|c|c|}
\hline USA & Puerto Rico & & & & & \\
\hline 5 & ex.com & 1 & enex.com & omenex.com & nenex.co.nz & \\
\hline
\end{tabular}

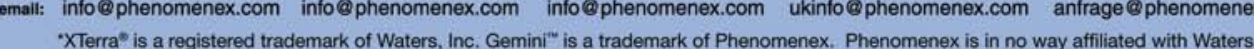
"XTerra" is a registered trademark of Waters, Inc. Gemini" is a trademark of Phenomenex. Phenomenex is in no way affiliated with Waters. 